\title{
Seagrass ecosystems status between the sliding baseline syndrome and the need for reference conditions
}

Montefalcone Monica $^{1 *}$, Vacchi Matteo ${ }^{2}$, Morri Carla ${ }^{1}$, Ferrari Marco ${ }^{1}$, Bianchi Carlo Nike ${ }^{1}$

1 DiSTAV, University of Genoa, Italy

${ }^{2}$ Aix-Marseille University, CEREGE CNRS-IRD UMR, Aix en Provence, France

*montefalcone@dipteris.unige.it

Marine coastal ecosystems are facing compositional and functional changes due to the increasing human footprint worldwide, and the assessment of their long-term changes becomes particularly challenging in this new "Anthropocene Epoch". Measures of change can be done by comparing the present ecosystem status to a defined baseline representing the reference condition. The "syndrome" of the sliding (or shifting) baselines, which describes the incremental lowering of ecological standards, has become a major concern when long-term changes have to be assessed because an already degraded environment status could be accepted as reference. The challenge of delineating ecosystems change in the context of sliding baselines is particularly relevant along highly anthropized coasts, as the case of the Liguria (NW Mediterranean Sea), where pristine coastal areas could not be expected anymore. The Marine Strategy Framework Directive (MSFD, 2008/56/EEC) suggests three approaches to define reference conditions: i) comparison with the status in Marine Protected Areas (MPAs); ii) use of historical information; iii) modelling. Here we report recent applications of these three approaches in Liguria to assess the status of seagrass meadow ecosystem. Ligurian MPAs are too recent and often insufficiently enforced, so that Posidonia oceanica meadows developing in MPAs are far from the expected good ecological status. Available historical information on seagrass distribution was sometime unreliable. Models are promising but face intellectual difficulties (e.g., choice of descriptors). Validating historical data with predictive models showed effective in delineating the trajectory of change experienced by the Ligurian seagrass meadows. Many meadows have been lost and most are showing structural degradation, which favoured the substitution by alien green algae of the genus Caulerpa and triggered a phase shift in the seagrass ecosystems, with the consequent loss of biodiversity, functioning and economic value of the ecosystem services provided by healthy meadows. 